\title{
Ocular autonomic dysfunction and intraocular pressure in leprosy
}

\author{
SUSAN LEWALLEN,' PAUL COURTRIGHT,' AND HO-SUNG LEE \\ From the Korea Eye Care Project, 'International Centre for Eye Health, 27-29 Cayton Street, London \\ ECIV 9EJ, and the 'Department of Ophthalmology, Catholic Hospital, Dae Myong dong 3065-6, Nam gu, \\ Daegu 705-034, South Korea
}

SUMmaRY We examined 241 leprosy patients and 135 age-matched healthy controls in central South Korea, measuring intraocular pressure in the supine and the upright positions and measuring the size of the pupils in darkness as an indication of ocular autonomic dysfunction. The mean intraocular pressure was significantly lower in the patients and the mean size of pupils was significantly smaller in the patients than in the controls. However, there was no correlation between pupil size and intraocular pressure in our patients. Our findings show that leprosy patients have ocular autonomic dysfunction, but do not support previous speculation that this dysfunction is the primary cause for low intraocular pressure in leprosy.

Leprosy is a disease which affects $10-12$ million people in the world.' There are wide variations in reports of the prevalence of ocular complications from it. ' Differences in race, type of disease, duration of disease, and reporting methodology may all be responsible for this variation. There are several ways in which the leprosy bacillus affects the eye. Lagophthalmos, superficial and interstitial keratitis, corneal nerve beading, and nodules on the sclera and cornea are manifestations of bacillary infiltration of nerves and anterior segment structures. Acute iridocyclitis may occur, but more frequent is a unique chronic iridocyclitis. In this condition the eye is white and quiet, with the gradual development of severe miosis. Posterior synechiae may develop as well as complicated cataracts.

Low intraocular pressure has been reported in leprosy patients in several studies. ${ }^{3.5}$ Furthermore, it has been reported that some leprosy patients show significant postural changes in intraocular pressure and suggested that this might be due to loss of autonomic function in the anterior segment of the eye, presumably due to bacillary infiltration of the ciliary nerves. ${ }^{5}$

We examined leprosy patients and healthy controls in a racially homogeneous population, looking for ocular pathology and measuring the intraocular

Correspondence to Dr Gordon J Johnson, Director, International Centre for Eye Health. pressure in the upright and supine positions. As a measure of ocular autonomic dysfunction we measured the size of the pupils in complete darkness. Size of the pupil in the dark has been shown to be a reliable measure of ocular autonomic dysfunction in diabetics. ${ }^{6}$ We present our findings in this paper.

\section{Materials and methods}

As part of a programme to train leprosy paramedical workers to recognise potentially blinding disease in leprosy patients all patients $(n=605)$ in eight leprosy resettlement villages in Kyeong Buk province, South Korea, were encouraged to undergo an ophthalmic examination. Of these patients $509(83 \%)$ were examined.

In small villages all examined patients were included in the intraocular pressure and pupil studies. In larger villages, for logistic reasons, a systematic sample of half the patients was selected for intraocular pressure and pupil study. Voluntary controls, known to be free of leprosy, were obtained from the outpatient department of the Catholic Skin Clinic and Hospital in Daegu. Controls were matched for age to the patient population. All subjects with diabetes and eyes with previous intraocular surgery or trauma were excluded.

Visual acuity was tested by a trained health worker. A sticker with an identification number was 
Table 1 Intraocular pressure and postural change in pressure in eyes of healthy controls and leprosy patients

\begin{tabular}{|c|c|c|c|c|c|c|}
\hline & \multicolumn{3}{|l|}{ Right eye } & \multicolumn{3}{|l|}{ Left eye } \\
\hline & Controls & Patients & $p$ & Controls & Patients & $p$ \\
\hline Intraocular pressure $(\mathrm{mm} \mathrm{Hg})$, mean with $\mathrm{SD}(\mathrm{no})$ & $14 \cdot 2(2 \cdot 9)(120)$ & $13 \cdot 6(3 \cdot 2)(218)$ & $0 \cdot 05$ & $14.2(2.9)(121)$ & $13 \cdot 1(3 \cdot 3)(216)$ & (0.01 \\
\hline Postural change in pressure $(\mathrm{mm} \mathrm{Hg})$, mean with SD (no) & $1 \cdot 4(2 \cdot 2)(118)$ & $1 \cdot 8(2 \cdot 8)(215)$ & $0 \cdot 15$ & $1.2(1.9)(119)$ & $1 \cdot 6(2 \cdot 3)(210)$ & ().16 \\
\hline
\end{tabular}

placed on the forehead between the eyebrows, and the patient was positioned against a head rest connected to the camera lens $(28 \mathrm{~mm}$ macro) and ring flash. A photograph of the patient's pupils was taken with 100 ASA slide film. With the patient in exactly the same position a darkness hood made of absolutely light-proof fabric was placed over the patient. The hood enclosed the camera lens and headrest and was designed to drape over the shoulders of the patient and exclude all light. After 20 seconds in complete darkness another photograph was taken. The patient was then examined by an ophthalmologist with a portable slit-lamp. Next the patient had one drop of fluorescein instilled in each eye and the intraocular pressure was measured in first the right, then the left eye with a Perkins applanation tonometer. The patient assumed the supine position and the intraocular pressure was immediately measured again, right eye then left eye.

The above procedure was followed on both patients and controls except that no visual acuity testing was done on controls. The slides of pupils were projected at a magnification of approximately $5 \times$, and the size of the pupils and the identification sticker in each slide was measured. An independent examiner read $10 \%$ of the slides to check agreement. Exact magnification of each slide was calculated from known and magnified sticker size and the true pupil diameter in $\mathrm{mm}$ calculated for each eye. All eyes with posterior synechiae were excluded when we analysed dark pupil size, since mechanical restriction of the pupil in these eyes would invalidate pupil size as a measure of autonomic dysfunction.

Relationships among variables (upright and supine intraocular pressure, pupil diameter, signs of ocular pathology, and age) were established for individual eyes by means of multiple regression analysis, Pearson's correlation coefficient ( $r$ ), and analysis of variance. To avoid problems caused by intracorrela- tion between right and left eye in an individual, we report results for left and right eyes separately. ${ }^{\text {? }}$

\section{Results}

We enrolled 241 leprosy patients in our study: 181 $(75 \%)$ had multibacillary disease and $53(22 \%)$ had paucibacillary disease. The remainder were of unknown disease classification. The age range was 28-76, mean 53 years. The mean duration of disease was 33 years. We enrolled 135 controls of similar age.

There was a significant difference between leprosy patients and healthy controls in intraocular pressures (Table 1). Furthermore, eyes of leprosy patients with posterior synechiae had a mean intraocular pressure of $12.6 \mathrm{~mm} \mathrm{Hg}$ compared with $13.5 \mathrm{~mm} \mathrm{Hg}$ in eyes of leprosy patients without synechiae $(p=0 \cdot 03)$. There was no association between age or disease type and intraocular pressure. The mean postural change in intraocular pressure was greater in patients than in controls but this difference was not statistically significant (Table 1).

The mean pupil size of leprosy patients was smaller than that of healthy controls (Table 2). In both leprosy patients and healthy controls size of pupils in the dark was age dependent. This dependence is shown in Fig. 1. (Since the left and right pupils are essentially the same size they have been combined in this figure.) Pupil size was not associated with disease type. There were few patients in the oldest age groups, resulting in increased variability of pupil size in these groups.

Among the patients' eyes there was a negative correlation (right eye $r=-0 \cdot 149$, left eye $r=-0 \cdot 117$ ) between intraocular pressure and postural changes in pressure $(p>0 \cdot 05)$. There was no association between either intraocular pressure or postural change in intraocular pressure and pupil size in the dark.

Table 2 Pupil size in the dark in eyes of healthy controls and leprosy patients

\begin{tabular}{|c|c|c|c|c|c|c|}
\hline & \multicolumn{3}{|l|}{ Right eye } & \multicolumn{3}{|l|}{ Left eye } \\
\hline & Controls & Patients & $p$ & Controls & Patients & $p$ \\
\hline Pupil size $(\mathrm{mm})$, mean with SD (no) & $5 \cdot 3(0 \cdot 07)(115)$ & $4 \cdot 8(1 \cdot 1)(182)$ & $0 \cdot(0) 1$ & $5 \cdot 4(0 \cdot 7)(117)$ & $4 \cdot 6(1 \cdot 3)(185)$ & (0.(x)1 \\
\hline
\end{tabular}


Fig. 1 Pupil size in the dark in leprosy patients (solid line) and healthy controls (broken line).

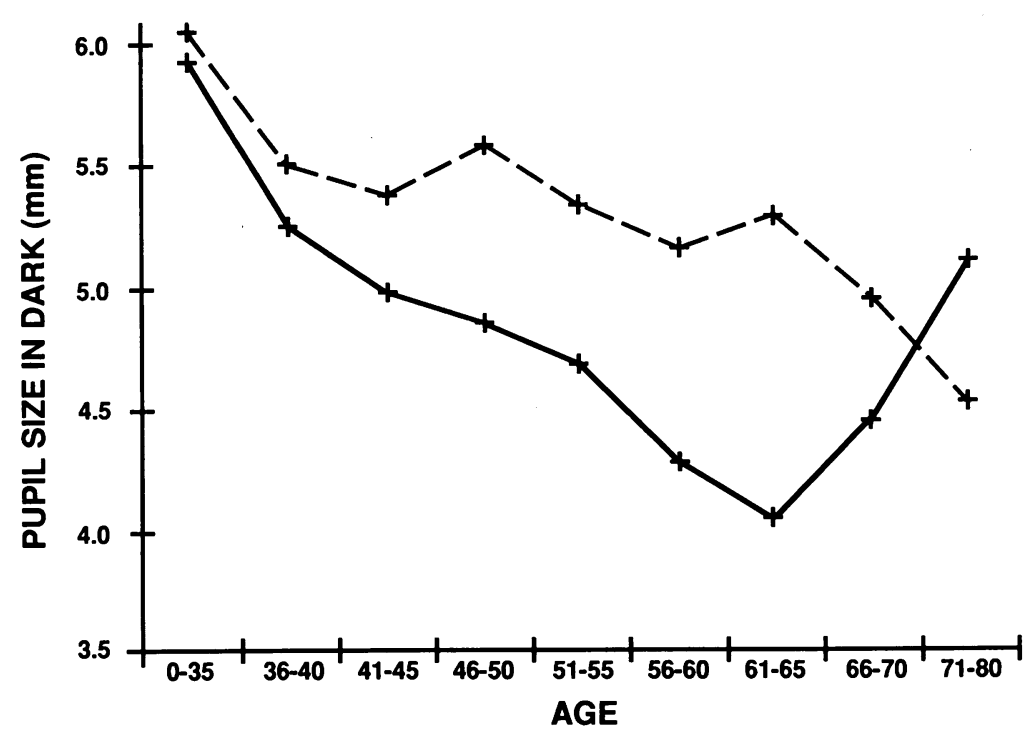

In the control group there was a positive correlation (right eye $r=0.237$, left eye $r=0 \cdot 267$ ) between intraocular pressure and pupil size in the dark $(p<0.01)$. There was no correlation between intraocular pressure and postural change in pressure.

\section{Discussion}

Our findings of intraocular pressure in leprosy patients confirm those of others - that is, pressures are lower in this group of patients than in the general population. We also demonstrate that leprosy patients show significant ocular autonomic dysfunction compared with normal controls. However, in our patients we did not find any association between ocular autonomic dysfunction and intraocular pressures or postural changes in intraocular pressure.

The mechanism by which the ocular autonomic system influences intraocular pressure is not well understood. We can only speculate as to the cause of lower intraocular pressure in leprosy patients. If these pressures were due only to ocular autonomic dysfunction, we would expect to find a correlation between dark pupil size and intraocular pressure in our patients, which we did not find. It was interesting that we did find such a correlation in our control group, in which decreasing sympathetic tone was associated with decreasing intraocular pressure independently of age.

Low intraocular pressures in leprosy could be due to damage to the ciliary body secondary to chronic low grade iridocyclitis. ffytche has suggested that the chronic iridocyclitis seen in multibacillary patients may be gradual atrophy of the dilator muscle (pre- sumably due to ocular sympathetic nerve dysfunction) rather than a true inflammatory process. ${ }^{\circ}$ Our findings support this theory in that we found that eyes of leprosy patients do not dilate normally in the dark in comparison with controls and that these eyes were white and quiet with no cells in the anterior chamber. In our study and others, ${ }^{3}$ leprosy patients with posterior synechiae have intraocular pressures significantly lower than patients without synechiae. However, our method of testing for autonomic dysfunction cannot be used in eyes with posterior synechiae, so it is not clear what the relative contributions of chronic iridocyclitis and ocular autonomic dysfunction to low intraocular pressure in leprosy might be.

As in a previous study, we found a negative correlation between intraocular pressure and postural change in pressure in our leprosy population; however this was not statistically significant in our study. The mechanisms controlling the homoeostasis of intraocular pressure with change in body position are not clear. There are reports that patients with open angle glaucoma, low tension glaucoma, ocular hypertension, systemic hypertension, and diabetes show large postural changes in intraocular pressure compared with normal persons. ${ }^{4-12}$ This phenomenon is thought to be related to vasomotor dysfunction. However, we found no association between postural change in intraocular pressure and ocular autonomic dysfunction.

It is desirable to find a method of detecting patients at risk for chronic iridocyclitis early in the disease before vision is impaired. Our findings do not suggest that intraocular pressure measurements will be help- 
ful in doing this. However, our leprosy patients all had a long disease duration. It is possible that control mechanisms which the autonomic system normally exerts over intraocular pressure may be severely altered in patients with long-standing disease. Longitudinal analysis of intraocular pressure in newly diagnosed patients will aid our understanding of this.

Studies of ocular autonomic function and intraocular pressure in leprosy patients will help clarify the ocular pathophysiology of this disease. Furthermore, studies of intraocular pressure in patients with ocular autonomic dysfunction may help to explain the role of the ocular autonomic nervous system in intraocular pressure regulation in healthy eyes.

This work was supported by the American Leprosy Missions. The authors thank the director and staff of the Catholic Skin Clinic and Hospital in Daegu for their assistance and to Dr H B Ostler and the Francis I Proctor Foundation for the use of equipment.

\section{References}

1 World Health Organisation Expert Committee on Leprosy. WHO Tech Rep Ser 768. Geneva, 1988: 9.

2 Courtright $P$. Defining the magnitude of ocular leprosy: problems of methodology. Int J Lepr Other Mycobact Dis 1988; 56: 566-73.
3 Brandt F, Malla OK, Anten JGF. Influence of untreated chronic plastic iridocyclitis on intraocular pressure in leprous patients. BrJ Ophthalmol 1981: 65: 240-2.

4 Slem G. Clinical studies of ocular leprosy. Am J Ophthalmol 1971: 71: 431-4.

5 Hussein N, Courtright P, Ostler HB, Hetherington J, Gelber $\mathrm{RH}$. Low intraocular pressure and postural changes in intraocular pressure in Hansen's disease patients. Am J Ophthalmol In press.

6 Smith SA, Dewhirst RD. A simple diagnostic test for pupillary abnormality in diabetic autonomic neuropathy. Diabetes Med 1986; 3: 38-41.

7 Newcombe RG, Duff GR. Eyes or patients? Traps for the unwary in the statistical analysis of ophthalmologic studies. Br J Ophthalmol 1987; 71: 645-6.

8 ffytche TJ. Role of iris changes as a cause of blindness in lepromatous leprosy. Br J Ophthalmol 1981; 65: 231-9.

9 Tsukahara S, Sasaki T. Postural change of IOP in normal persons and in patients with primary open-angle glaucoma and lowtension glaucoma. Br J Ophthalmol 1984; 68: 383-8.

10 Krieglstein GK, Langham ME. Influence of body position and the intraocular pressure of normal and glaucomatous eyes. Ophthalmologica 1975; 171: 132-45.

11 Leonard TJ, Kerr Muir MG, Kirby GR, Hitchings RA. Ocular hypertension and posture. Br J Ophthalmol 1983; 67: 362-6.

12 Williams BI, Peart WS, Letley E. Abnormal intraocular pressure control in systemic hypertension and diabetes mellitus. BrJ Ophthalmol 1980; 64: 84.5-51.

Accepted for publication 30 June 1989. 